Stella Piro-Vernier · Francesco Ragnedda · Vincenzo Vespri

\title{
Hölder regularity for bounded solutions to a class of anisotropic operators
}

Received: 25 November 2017 / Accepted: 22 April 2018 / Published online: 2 May 2018

Abstract. In this note we show the Hölder regularity for bounded solutions to a class of anisotropic elliptic operators. This result is the dual of the one proved by Liskevich and Skrypnik (Nonlinear Anal 71:1699-1708, 2009).

\section{Introduction}

In this paper we consider the following class of anisotropic equations

$$
\sum_{i=1}^{N-1} \frac{\partial}{\partial x_{i}} A_{q, i}(x, u, D u)+\frac{\partial}{\partial x_{N}} A_{p}(x, u, D u)=0 \text { in } \Omega,
$$

with $\Omega$ a regular domain in $\mathbb{R}^{N}, p>q>1$. The functions $A_{q, i}(x, u, D u)$ and $A_{p}(x, u, D u): \Omega \times \mathbb{R}^{N+1} \rightarrow \mathbb{R}^{N}$ are assumed to be measurable and satisfying the structure conditions

$$
\begin{cases}\text { i) } & \sum_{i=1}^{N-1} A_{q, i}(x, u, \eta) \cdot \eta_{i} \geq C_{0}\left|\eta^{\prime}\right|^{q} \\ \text { ii }) & A_{p}(x, u, \eta) \cdot \eta_{N} \geq C_{0}\left|\eta_{N}\right|^{p} \\ \text { iii }) & \sum_{i=1}^{N-1}\left|A_{q, i}(x, u, \eta)\right| \leq C_{1}\left|\eta^{\prime}\right|^{q-1} \\ \text { iv }) \quad\left|A_{p}(x, u, \eta)\right| \leq C_{1}\left|\eta_{N}\right|^{p-1},\end{cases}
$$
$R^{N}$

where $C_{0}, C_{1}$ are positive constants and for any vector $\eta=\left(\eta_{1}, \ldots, \eta_{N}\right)$ in

$$
\eta^{\prime}=\left(\eta_{1}, \ldots, \eta_{N-1}\right)
$$

S. Piro-Vernier $(\bowtie)$. F. Ragnedda: Dipartimento di Matematica e Informatica, Università di Cagliari, Viale Merello 92, 09123 Cagliari, Italy. e-mail: svernier@unica.it; ragnedda@unica.it

V. Vespri: Dipartimento di Matematica “U. Dini”, Università di Firenze, Viale Morgagni 67/A, 50134 Florence, Italy. e-mail: vespri@math.unifi.it

Mathematics Subject Classification: 35J70 · 35J92 - 35B65; Secondary 35B45 
The prototype of this class of operators is the anisotropic p-Laplacean:

$$
\sum_{i=1}^{N-1} \frac{\partial}{\partial x_{i}}\left(\left|\frac{\partial u}{\partial x_{i}}\right|^{q-2} \frac{\partial u}{\partial x_{i}}\right)+\frac{\partial}{\partial x_{N}}\left(\left|\frac{\partial u}{\partial x_{N}}\right|^{p-2} \frac{\partial u}{\partial x_{N}}\right)=0 \text { in } \Omega .
$$

Let us introduce two functional spaces:

$$
\begin{aligned}
& W^{1,[q, p]}(\Omega)=\left\{u \in L^{1}(\Omega): D_{x_{i}} u \in L^{q}(\Omega), i=1, \ldots, N-1 ; D_{x_{N}} u \in L^{p}(\Omega)\right\} \\
& W_{0}^{1,[q, p]}(\Omega)=W^{1,[q, p]}(\Omega) \cap W_{0}^{1,1}(\Omega) .
\end{aligned}
$$

Definition. A function $u \in W_{l o c}^{1,[q, p]}(\Omega)$ is a local, weak solution to (1) if for every compact set $K \subset \Omega$

$$
\int_{K} \sum_{i=1}^{N-1} A_{q, i}(x, u, D u) \frac{\partial \varphi}{\partial x_{i}} d x+\int_{K} A_{p}(x, u, D u) \frac{\partial \varphi}{\partial x_{N}} d x=0
$$

for all test functions $\varphi \in W_{0}^{1,[q, p]}(\Omega)$, where $\varphi$ has compact support in $K$.

In the last decades many papers were devoted to the study on the anisotropic operators (see, for instance [1-3,17,18]) but the question of the Hölder regularity of solutions to equations with measurable coefficients was left open (see, for instance, the survey paper [19]).

A first step to face this problem was made by Liskevich and Skrypnik [15] in 2009. They considered the following class of anisotropic quasilinear equations

$$
\frac{\partial^{2} u}{\partial x_{1}^{2}}+\sum_{i=2}^{N} \frac{\partial}{\partial x_{i}} A_{i}(x, u, D u)+b(x, u, D u)=0 \quad \text { in } \Omega .
$$

They assumed $p>2$, a suitable condition on the lower terms, the following structure conditions

$$
\left\{\begin{array}{l}
\text { i) } \quad \sum_{i=2}^{N} A_{i}(x, u, \eta) \cdot \eta_{i} \geq C_{0}|\bar{\eta}|_{N-1}^{p} \\
\text { ii }) \quad\left|A_{i}(x, u, \eta)\right| \leq C_{1}|\bar{\eta}|_{N-1}^{p-1},
\end{array}\right.
$$

where $\bar{\eta}=\left(\eta_{2}, \ldots, \eta_{N}\right)$ and the following nearness between the exponents holds:

$$
2<p \leq \frac{N \bar{p}}{N-\bar{p}}, \quad \frac{1}{\bar{p}}=\frac{1}{N}\left(\frac{1}{2}+\frac{N-1}{p}\right), \quad \bar{p}<N .
$$

We recall that this condition implies the local boundedness of the solutions (see $[13,16])$.

More precisely they realized that the parabolic techniques (the so called time expansion of positivity techniques) developed in [7] to study the degenerate equation

$$
u_{t}=\Delta_{p} u, \quad p>2
$$


can be adapted to study the elliptic case too, by proving a suitable expansion of positivity in space variables.

In [11], the result proved in [15] was extended to the following class of anisotropic equations

$$
\frac{\partial}{\partial x_{1}} A_{p}(x, u, D u)+\sum_{i=2}^{N} \frac{\partial}{\partial x_{i}} A_{q, i}(x, u, D u)=0 \text { in } \Omega \text {. }
$$

The authors assumed $q>p>1$, the structure conditions

$$
\begin{cases}\text { i) } \quad & A_{p}(x, u, \eta) \cdot \eta_{1} \geq C_{0}\left|\eta_{1}\right|^{p} \\ \text { ii }) \quad & \left|A_{p}(x, u, \eta)\right| \leq C_{1}\left|\eta_{1}\right|^{p-1} \\ \text { iii }) & \sum_{i=2}^{N} A_{q, i}(x, u, \eta) \cdot \eta_{i} \geq C_{0}|\bar{\eta}|^{q} \\ \text { iv }) & \left|A_{q, i}(x, u, \eta)\right| \leq C_{1}|\bar{\eta}|^{q-1}\end{cases}
$$

and the local boundness of $u$. The last assumption is alternative to the assumption of the nearness of $p$ and $q$.

The approach of the space expansion of positivity seems very promising and it works in different context (see [10,12]).

More recently, in [9], a stability result was proved.

Let $u$ be a locally bounded weak solution $u$ of the equation

$$
\sum_{i=1}^{N} D_{x_{i}}\left(A_{p_{i}}(x, u, D u)\right)=0 \text { in } \Omega .
$$

Assume $p_{i}>1, i=1, \ldots, N$, their harmonic mean $\bar{p}$ less than the dimension of the space $N$ and the following structure conditions:

$$
\left\{\begin{array}{l}
\text { i) } \quad \sum_{i=1}^{N} A_{p_{i}}(x, u, D u) \cdot D_{x_{i}} u \geq C_{0} \sum_{i=1}^{N}\left|D_{x_{i}} u\right|^{p_{i}}, \\
\text { ii }) \quad\left|A_{p_{i}}(x, u, D u)\right| \leq C_{1}\left|D_{x_{i}} u\right|^{p_{i}-1}, \quad \forall i=1, \ldots, N .
\end{array}\right.
$$

There exists a $q_{0}>1$ such that if

$$
p_{\max }-p_{\min } \leq 1 / q_{0},
$$

with $p_{\min }=\min \left\{p_{1}, \ldots, p_{N}\right\}$ and $p_{\max }=\max \left\{p_{1}, \ldots, p_{N}\right\}$, then the solution is locally Hölder continuous in $\Omega$.

The aim of the present paper is to give a new insight of this difficult, challenging and still open problem. Here we prove, in a certain sense, a dual result with respect the one proved in $[11,15]$. This should be an important step to prove the regularity in the physical case of 3 spatial dimensions having each direction a different diffusion exponent. 
As in [11], the condition on the nearness of the exponents does not appear. Perhaps, such a condition is necessary for the boundedness of the solutions but it does not play any role for the regularity of already bounded solutions.

As in $[11,15]$, the proof is based on a suitable space expansion of positivity, but, differently from the quoted papers, here one can avoid to work with the transformed equation and this makes simpler the calculations.

In the sequel we say that a constant depends only upon the data if it depends only upon $N, C_{0}, C_{1}, p, q$ and $\|u\|_{L^{\infty}(\Omega)}$.

Theorem 1.1. Let $u(x)$ be a locally bounded weak solution to (1)-(2) with $p>$ $q>1$, then $u(x)$ is locally Hölder continuous.

More precisely, in analogy with the parabolic p-Laplacean, it is possible to prove that there are two positive constants $\beta<1$ and $C$ depending only upon the data such that for any compact $K$ strictly contained in $\Omega$ and for any $P_{1}=\left(x^{\prime}, x_{N}\right)$, $P_{2}=\left(y^{\prime}, y_{N}\right) \in K$

$$
\left|u\left(P_{1}\right)-u\left(P_{2}\right)\right| \leq C\|u\|_{L_{\infty}(\Omega)}\left(\frac{\left|x_{N}-y_{N}\right|+\|u\|_{L_{\infty}(\Omega)}^{\frac{p-q}{p}}\left|x^{\prime}-y^{\prime}\right|^{\frac{q}{p}}}{(p, q)-\operatorname{dist}(K, \partial \Omega)}\right)^{\beta},
$$

where $(p, q)-\operatorname{dist}(K, \partial \Omega)$ is the infimum of $\left|x_{N}-y_{N}\right|+\|u\|_{L_{\infty}(\Omega)}^{\frac{p-q}{p}}\left|x^{\prime}-y^{\prime}\right|^{\frac{q}{p}}$ ranging $\left(x^{\prime}, x_{N}\right) \in K$ and $\left(y^{\prime}, y_{N}\right) \in \partial \Omega$. Such a proof is quite technical and, nowadays, standard. For these reason we omit it and we refer the reader to Chapter 3 of [5].

In our forthcoming researches, as already written, we intend to combine this new approach with the ideas developed in $[11,15]$ to prove the regularity of the solutions under more general assumptions.

The scheme of this paper is the following: in Sect. 2 we state some preliminary results. In Sect. 3 we begin to prove the main Theorem starting an alternative argument that we conclude in Sect. 4 . The proof of Theorem 1.1 will result a direct consequence of the alternative argument.

\section{Preliminary results}

In this section we collect some results we will use in the sequel.

Let $r>0$ and define a ball in the last $N-1$ variables

$$
\bar{B}_{r}=\left\{\left(x_{2}, \ldots, x_{N}\right) \in \mathbb{R}^{N-1}: \sum_{i=2}^{N} x_{i}^{2} \leq r^{2}\right\},
$$

and define the following cylinder in $\Omega$,

$$
Q_{L, r}=\left\{x \in \mathbb{R}^{N}:\left|x_{1}\right| \leq L,\left(x_{2}, \ldots, x_{N}\right) \in \bar{B}_{r}\right\} .
$$

Assume $Q_{1}:=Q_{L, r}$ contained in $\Omega$. Consider another cylinder $Q_{2}:=Q_{L_{1}, r_{1}}$ with $L_{1}<L$ and $r_{1}<r$. 
Denote with $\omega$ the oscillation of $u$ in $\Omega$ and let $\mu_{-}$be the ess inf of $u$ in $\Omega$. Define

$$
G(u):=\left[\frac{1}{\left(u-\mu_{-}+a \omega H\right)^{q-1}}-\frac{1}{(\omega H)^{q-1}}\right]_{+},
$$

where $[\cdot]_{+}$denotes the positive part, thus $G(u)=0$ if $u>\mu_{-}+(1-a) \omega H$ and $G(u)$ is positive a.e. if $u \leq \mu_{-}+(1-a) \omega H$. Here $0<a<1,0<H<1$.

Lemma 2.1. Let $u$ be a locally bounded weak solution of equation (1) and assume that the structure conditions (2) are satisfied. Then there exists a constant $C>0$ (depending only upon the data) such that

$$
\begin{aligned}
& \int_{Q_{2} \cap A}\left|D_{x_{1}} \ln _{+} \frac{H \omega}{u-\mu_{-}+a \omega H}\right|^{q} d x \leq C \int_{Q_{1} \cap A}\left|D_{x} \xi\right|^{q} d x \\
& +C \int_{Q_{1} \cap A}\left|D_{x_{N}} \xi\right|^{p}\left(u-\mu_{-}+a \omega H\right)^{p-q} d x
\end{aligned}
$$

where $D_{x}$ is the gradient in the first $N-1$ variables, $A=\left\{x \in \Omega: u<\mu_{-}+(1-\right.$ a) $\omega H\}$ and $\xi \in C^{\infty}$ is a function such that $\xi=1$ in $Q_{2}, \xi=0$ in $\Omega \backslash Q_{1}$.

Proof. Let us take $G(u) \xi^{p}$ as a test function. Then using equation (1), we have

$$
\int_{\Omega} \sum_{i=1}^{N-1} A_{q, i}(x, u, D u) D_{x_{i}}\left(G(u) \xi^{p}\right) d x+\int_{\Omega} A_{p}(x, u, D u) D_{x_{N}}\left(G(u) \xi^{p}\right) d x=0 .
$$

If we use the definition of $G(u)$ and condition (2), then

$$
\begin{aligned}
& (q-1) C_{0} \int_{Q_{1} \cap A}\left|D_{x} u\right|^{q} \frac{1}{\left(u-\mu_{-}+a \omega H\right)^{q}} \xi^{p} d x \\
& +(q-1) C_{0} \int_{Q_{1} \cap A}\left|D_{x_{N}} u\right|^{p} \frac{1}{\left(u-\mu_{-}+a \omega H\right)^{q}} \xi^{p} d x \\
& \leq p C_{1} \int_{Q_{1} \cap A}\left|D_{x} u\right|^{q-1} \frac{1}{\left(u-\mu_{-}+a \omega H\right)^{q-1}} \xi^{p-1}\left|D_{x} \xi\right| d x+ \\
& +p C_{1} \int_{Q_{1} \cap A}\left|D_{x_{N}} u\right|^{p-1} \frac{1}{\left(u-\mu_{-}+a \omega H\right)^{q-1}} \xi^{p-1}\left|D_{x_{N}} \xi\right| d x
\end{aligned}
$$

The estimate (9) follows by using Young inequality in the right hand side of the previous estimate. 
Lemma 2.2. (Sobolev-Troisi Inequality [21], see also [20] and [14]) Let $\Omega \subset \mathbb{R}^{N}$ be a bounded open set and consider $u \in W_{0}^{1,\left[p_{1}, \ldots, p_{N}\right]}(\Omega), p_{i} \geq 1$ for all $i=$ $1, \ldots, N$. Let

$$
\frac{1}{\bar{p}}=\frac{1}{N} \sum_{i=1}^{N} \frac{1}{p_{i}}, \quad \bar{p}^{*}=\frac{N \bar{p}}{N-\bar{p}} .
$$

Then there exists $c$ depending on $N, p_{1}, \ldots, p_{N}$ if $\bar{p}<N$ such that

$$
\|u\|_{L_{p^{*}}(\Omega)}^{N} \leq c \prod_{i=1}^{N}\left\|D_{x_{i}} u\right\|_{L_{p_{i}}(\Omega)} .
$$

Lemma 2.3. (Algebraical lemma [4], see also [8]) Let $\left\{Y_{m}\right\}, m=0,1,2, \ldots$, be a sequence of positive numbers, satisfying the recursive inequalities

$$
Y_{m+1} \leq C b^{m} Y_{m}^{1+\lambda}
$$

where $C, b>1$ and $\lambda>0$ are given numbers. If

$$
Y_{0} \leq C^{\frac{-1}{\lambda}} b^{\frac{-1}{\lambda^{2}}}
$$

then $\left\{Y_{m}\right\}$ converges to zero as $m \rightarrow \infty$.

Lemma 2.4. (Generalised Caccioppoli's Inequality) Let $u$ be a locally bounded weak solution of (1) and assume that the structure conditions in (2) are satisfied. Then there exists a constant $C>0$ (depending only upon the data) such that for every test function $\theta \in C_{0}^{1}(\Omega)$, we have

$$
\begin{aligned}
& \int_{\Omega} \theta^{p}\left(\left|D_{x}(u-k)_{-}\right|^{q}+\left|D_{x_{N}}(u-k)_{-}\right|^{p}\right) d x \\
& \leq C \int_{\Omega}\left(\theta^{p-q}\left|D_{x} \theta\right|^{q}\left|(u-k)_{-}\right|^{q}+\left|D_{x_{N}} \theta\right|^{p}\left|(u-k)_{-}\right|^{p}\right) d x,
\end{aligned}
$$

where $D_{x}$ is the gradient in the first $N-1$ variables and $k$ any positive constant.

Note that an analogous result also holds if we deal with $(u-k)_{+}$.

Proof. Take

$$
\psi=\psi(x)=-\theta(x)^{p}(u-k)_{-}, \quad \theta \in C_{0}^{1}(\Omega) .
$$

as a test function. Note that

$$
\begin{aligned}
D_{x} \psi & =p \theta^{p-1}(u-k)_{-} D_{x} \theta+\theta^{p} D_{x}(u-k)_{-} \\
D_{x_{N}} \psi & =p \theta^{p-1}(u-k)_{-} D_{x_{N}} \theta+\theta^{p} D_{x_{N}}(u-k)_{-} .
\end{aligned}
$$


Then using Eq. (1), we have

$$
\begin{aligned}
& \int_{\Omega} p \theta^{p-1}(u-k)_{-} \sum_{i=1}^{N-1} A_{q, i}(x, u, D u) D_{x_{i}} \theta d x \\
& +\int_{\Omega} \theta^{p} \sum_{i=1}^{N-1} A_{q, i}(x, u, D u) D_{x_{i}}(u-k)_{-} d x \\
& \quad+\int_{\Omega} p \theta^{p-1}(u-k)_{-} A_{p}(x, u, D u) D_{x_{N}} \theta d x \\
& +\int_{\Omega} \theta^{p} A_{p}(x, u, D u) D_{x_{N}}(u-k)_{-} d x=0 .
\end{aligned}
$$

If we take into account (2), we obtain the following inequality

$$
\begin{aligned}
& C_{0} \int_{\Omega} \theta^{p}\left(\left|D_{x}(u-k)_{-}\right|^{q}+\left|D_{x_{N}}(u-k)_{-}\right|^{p}\right) d x \\
& \leq p C_{1} \int_{\Omega}(N-1) \theta^{p-1}\left|(u-k)_{-}\right|\left|D_{x} \theta\right|\left|D_{x}(u-k)_{-}\right|^{q-1} d x \\
& \quad+p C_{1} \int_{\Omega} \theta^{p-1}\left|(u-k)_{-}\right|\left|D_{x_{N}} \theta\right|\left|D_{x_{N}}(u-k)_{-}\right|^{p-1} d x .
\end{aligned}
$$

Again, the statement follows by applying Young inequality.

In the next lemma we will consider a DeGiorgi type lemma. It is necessary to introduce the intrinsic geometry induced by the anisotropy of the operator itself (for more details about the intrinsic geometry see [5,22]).

For a Lebesgue measurable set $E \subset \mathbb{R}^{N}$ denote by $|E|$ its measure.

Let $R_{0}, k_{0}>0$ be given numbers and $R_{N}=R_{0}^{\frac{q}{p}} k_{0}^{\frac{p-q}{p}}$. Define for $j=$ $0,1,2, \ldots$

$$
Q_{R_{0}, j}:=\left\{x:\left|x_{i}\right|<R_{j}, \forall i=1, \ldots, N-1,\left|x_{N}\right|<r_{j}\right\},
$$

where $R_{j}=\frac{R_{0}}{2}+\frac{R_{0}}{2^{j+1}}$ and $r_{j}=\frac{R_{N}}{2}+\frac{R_{N}}{2^{j+1}}$.

Let $c_{j}=\left|Q_{R_{0}, j}\right|$ the measure of the parallelepiped $Q_{R_{0}, j}$.

Clearly $c_{j}$ is a decreasing sequence converging to a strictly positive constant $c_{\infty}$

Let $u$ be a bounded weak solution of (1) in $Q_{R_{0}, 0}$, assume that the structure conditions in (2) are satisfied and let $\mu_{+}=\sup _{Q_{R_{0}, 0}} u$ and $\mu_{-}=\inf _{Q_{R_{0}, 0}} u$.

Define

$$
A_{s, j}:=\left\{x \in Q_{R_{0}, s}: u(x) \leq k_{j}\right\}, \quad k_{j}=\frac{k_{0}}{2}+\frac{k_{0}}{2^{j+1}}+\mu_{-} .
$$

and $Z_{j}:=\frac{\left|A_{2 j, j}\right|}{\left|Q_{R_{0}, 2 j}\right|}$ 
Let denote $A_{\infty}$ as the intersection of the sets $A_{2 j, j}$, i.e,

$$
A_{\infty}=\bigcap_{j} A_{2 j, j}=\left\{x \in Q_{R_{\infty}}: u(x) \leq \frac{k_{0}}{2}+\mu_{-}\right\} .
$$

Lemma 2.5. (DeGiorgi type lemma) Let u be a bounded weak solution of (1) in $Q_{R_{0}, 0}$, assume that the structures conditions in (2) are satisfied. There is a number $v>0$ depending only upon the data (but not depending on $u, R_{0}$, and $k_{0}$ ) such that if $Z_{0}<v$ then $\left\{Z_{j}\right\}$ converges to zero as $j$ goes to infinity.

Proof. Let $\bar{p}$ and $\bar{p}^{*}$ be defined as in Sobolev-Troisi Lemma 2.2. If we consider the set $A_{2 j+2, j+1}$, we can write

$$
\int_{A_{2 j+2, j+1}}\left|u-k_{j}\right|^{\bar{p}} d x \geq\left(k_{j}-k_{j+1}\right)^{\bar{p}}\left|A_{2 j+2, j+1}\right| .
$$

Then

$$
\begin{aligned}
\left|A_{2 j+2, j+1}\right| & \leq \frac{1}{\left(k_{j}-k_{j+1}\right)^{\bar{p}}} \int_{A_{2 j+2, j+1}}\left|\left(u-k_{j}\right)_{-}\right|^{\bar{p}} d x \\
& \leq \frac{1}{\left(k_{j}-k_{j+1}\right)^{\bar{p}}} \int_{A_{2 j+1, j}} \theta_{2 j+1}^{\bar{p}}\left|\left(u-k_{j}\right)_{-}\right|^{\bar{p}} d x .
\end{aligned}
$$

Here $\theta_{2 j+1} \in C_{\infty}$ is a function such that $\theta_{2 j+1}=1$ in $Q_{R_{0}, 2 j+2}$ and $\theta_{2 j+1}=0$ out of $Q_{R_{0}, 2 j+1}$ satisfying $\left|D_{x} \theta_{2 j+1}(z)\right| \leq \tilde{c} \frac{4^{j}}{R_{0}}$, (where we recall once again that $D_{x}$ is the gradient with respect the first $N-1$ variables), $\left|D_{x_{N}} \theta_{2 j+1}(z)\right| \leq \tilde{c} \frac{4^{j}}{R_{N}}$ for a positive constant $\tilde{c}$ and for all $z \in Q_{R_{0}, 2 j+1}$.

If we use Hölder inequality, we obtain

$$
\left|A_{2 j+2, j+1}\right| \leq \frac{1}{\left(k_{j}-k_{j+1}\right)^{\bar{p}}}\left[\int_{A_{2 j+1, j}}\left(\theta_{2 j+1}\left|\left(u-k_{j}\right)_{-}\right|\right)^{\bar{p}^{*}} d x\right]^{\frac{\bar{p}}{\bar{p}^{*}}}\left|A_{2 j+1, j}\right|^{1-\frac{\bar{p}}{\bar{p}^{*}}},
$$

Now by Lemma 2.2, we have

$$
\begin{aligned}
\left|A_{2 j+2, j+1}\right| \leq & c \frac{1}{\left(k_{j}-k_{j+1}\right)^{\bar{p}}}\left|A_{2 j+1, j}\right|^{1-\frac{\bar{p}}{\bar{p}^{*}}} \\
& \prod_{i=1}^{N-1}\left[\int_{A_{2 j+1, j}}\left|D_{x_{i}}\left(\theta_{2 j+1}\left(u-k_{j}\right)_{-}\right)\right|^{q} d x\right]^{\frac{\bar{p}}{q N}} \\
& {\left[\int_{A_{2 j+1, j}}\left|D_{x_{N}}\left(\theta_{2 j+1}\left(u-k_{j}\right)_{-}\right)\right|^{p} d x\right]^{\frac{\bar{p}}{p^{N}}} }
\end{aligned}
$$




$$
\begin{aligned}
\leq & c \frac{1}{\left(k_{j}-k_{j+1}\right)^{\bar{p}}}\left|A_{2 j+1, j}\right|^{1-\frac{\bar{p}}{\bar{p}^{*}}} \\
& {\left[\int_{A_{2 j+1, j}}\left|D_{x}\left(\theta_{2 j+1}\left(u-k_{j}\right)_{-}\right)\right|^{q} d x\right]^{\frac{(N-1) \bar{p}}{N q}} } \\
& {\left[\int_{A_{2 j+1, j}}\left|D_{x_{N}}\left(\theta_{2 j+1}\left(u-k_{j}\right)_{-}\right)\right|^{p} d x\right]^{\frac{\bar{p}}{p N}} }
\end{aligned}
$$

Let us estimate $\int_{A_{2 j+1, j}}\left|D_{x}\left(\theta_{2 j+1}\left(u-k_{j}\right)_{-}\right)\right|^{q} d x$. We have

$$
\begin{aligned}
& \int_{A_{2 j+1, j}}\left|D_{x}\left(\theta_{2 j+1}\left(u-k_{j}\right)_{-}\right)\right|^{q} d x \\
& \leq C \int_{A_{2 j+1, j}}\left(\left|D_{x}\left(\theta_{2 j+1}\right)\right|^{q}\left|\left(u-k_{j}\right)_{-}\right|^{q}+\theta_{2 j+1}^{q}\left|D_{x}\left(u-k_{j}\right)_{-}\right|^{q}\right) d x .
\end{aligned}
$$

Considering the properties of the function $\theta_{2 j+1}$, we have

$$
\begin{aligned}
\int_{A_{2 j+1, j}}\left|D_{x}\left(\theta_{2 j+1}\left(u-k_{j}\right)_{-}\right)\right|^{q} d x \leq & C \frac{4^{j q}}{R_{0}^{q}} \int_{A_{2 j+1, j}}\left|\left(u-k_{j}\right)_{-}\right|^{q} d x \\
& +C \int_{A_{2 j+1, j}}\left|D_{x}\left(u-k_{j}\right)_{-}\right|^{q} d x .
\end{aligned}
$$

If we pass to the larger set $A_{2 j, j}$ and use the function $\theta_{2 j}$ with exponent $p$, we obtain

$$
\begin{aligned}
\int_{A_{2 j+1, j}}\left|D_{x}\left(\theta_{2 j+1}\left(u-k_{j}\right)_{-}\right)\right|^{q} d x \leq & C \frac{4^{j q}}{R_{0}^{q}} \int_{A_{2 j, j}}\left|\left(u-k_{j}\right)_{-}\right|^{q} d x \\
& +C \int_{A_{2 j, j}} \theta_{2 j}^{p}\left|D_{x}\left(u-k_{j}\right)_{-}\right|^{q} d x .
\end{aligned}
$$

Here we can apply Caccioppoli's Inequality of Lemma 2.4 to the second integral on the right hand side and we get, 


$$
\begin{aligned}
\int_{A_{2 j+1, j}}\left|D_{x}\left(\theta_{2 j+1}\left(u-k_{j}\right)_{-}\right)\right|^{q} d x \leq & C \frac{4^{j q}}{R_{0}^{q}} \int_{A_{2 j, j}}\left|\left(u-k_{j}\right)_{-}\right|^{q} d x \\
& +C \int_{A_{2 j, j}}\left|D_{x} \theta_{2 j}\right|^{q}\left|\left(u-k_{j}\right)_{-}\right|^{q} d x \\
& +C \int_{A_{2 j, j}}\left|D_{x_{N}} \theta_{2 j}\right|^{p}\left|\left(u-k_{j}\right)_{-}\right|^{p} d x .
\end{aligned}
$$

If we use the properties of $\theta_{2 j}$,

$$
\begin{aligned}
\int_{A_{2 j+1, j}}\left|D_{x}\left(\theta_{2 j+1}\left(u-k_{j}\right)_{-}\right)\right|^{p} d x \leq & C \frac{4^{j q}}{R_{0}^{q}} \int_{A_{2 j, j}}\left|\left(u-k_{j}\right)_{-}\right|^{q} d x \\
& +C \frac{4^{j p}}{R_{N}^{p}} \int_{A_{2 j, j}}\left|\left(u-k_{j}\right)_{-}\right|^{p} d x .
\end{aligned}
$$

Noting that $\left|u-k_{j}\right| \leq 2 k_{0}$ and by the definition of $R_{N}$ we have

$$
\int_{A_{2 j+1, j}}\left|D_{x}\left(\theta_{2 j+1}\left(u-k_{j}\right)_{-}\right)\right|^{p} d x \leq C \frac{4^{j p}}{R_{0}^{q}} k_{0}^{q}\left|A_{2 j, j}\right|
$$

Arguing as above, one obtains a similar estimate also for

$$
\int_{A_{2 j+1, j}}\left|D_{x_{N}}\left(\theta_{2 j+1}\left(u-k_{j}\right)_{-}\right)\right|^{p} d x
$$

i.e.

$$
\begin{aligned}
\int_{A_{2 j+1, j}}\left|D_{x_{N}}\left(\theta_{2 j+1}\left(u-k_{j}\right)_{-}\right)\right|^{p} d x \leq & C \frac{4^{j q}}{R_{0}^{q}} \int_{A_{2 j, j}}\left|\left(u-k_{j}\right)_{-}\right|^{q} d x \\
& +C \frac{4^{j p}}{R_{N}^{p}} \int_{A_{2 j, j}}\left|\left(u-k_{j}\right)_{-}\right|^{p} d x \\
\leq & C \frac{4^{j p}}{R_{0}^{q}} k_{0}^{q}\left|A_{2 j, j}\right| .
\end{aligned}
$$

Then since $\frac{\bar{p}(N-1)}{q N}+\frac{\bar{p}}{p N}=1$ we obtain from (10),

$$
\left|A_{2 j+2, j+1}\right| \leq C \frac{1}{\left(k_{j}-k_{j+1}\right)^{\bar{p}}}\left|A_{2 j+1, j}\right|^{1-\frac{\bar{p}}{\bar{p}^{*}}}\left[\frac{4^{j p}}{R_{0}^{q}} k_{0}^{q}\left|A_{2 j, j}\right| .\right] .
$$


Noting that $\left|A_{2 j+1, j}\right| \leq\left|A_{2 j, j}\right|$ we get from (11) that,

$$
\left|A_{2 j+2, j+1}\right| \leq \frac{2^{(j+2) \bar{p}}}{k_{0}^{\bar{p}}}\left|A_{2 j, j}\right|^{1+\left(1-\frac{\bar{p}}{\left.\bar{p}^{*}\right)}\right.} 4^{(j+1) p} \frac{k_{0}^{q}}{R_{0}^{q}} .
$$

Divide both sides by $\left|Q_{R_{0}, 2 j}\right|$ to have the following inequality

$$
Z_{j+1} \leq C 2^{(j+2) \bar{p}} 4^{(j+1) p} Z_{j}^{1+\lambda}
$$

where $\lambda:=\left(1-\frac{\bar{p}}{\bar{p}^{*}}\right)$.

Then the required statement follows by Lemma 2.3 .

In the sequel we need to apply the DeGiorgi type lemma in a very special case that allows us to get rid of the anisotropy. More specifically: consider a cube of side $2 R_{0}$, i.e

$$
Q_{R_{0}}:=\left\{x:\left|x_{i}\right| \leq R_{0}, \forall i=1, \ldots, N\right\} .
$$

Let $u$ be a bounded weak solution of (1) in $Q_{R_{0}}$, assume that the structure conditions in (2) are satisfied and let $\mu_{+}=\sup _{Q_{R_{0}}} u$ and $\mu_{-}=\inf Q_{R_{0}} u$.

Assume that

$$
u\left(x_{1}, \ldots, x_{N}\right) \geq \mu_{-}+k_{0},
$$

when $\left|x_{i}\right| \leq R_{0}$ for $i=1, \ldots, N-1$ and $x_{N}= \pm R_{0}$.

Lemma 2.6. (When the anisotropic DeGiorgi Lemma becomes isotropic) Let $u$ be a bounded weak solution of (1) in $Q_{R_{0}}$, assume that conditions (2) and (12) are satisfied. Then there is a number $v>0$ depending only upon the data (but not depending on $u, R_{0}$, and $\left.k_{0}\right)$ and a constant $\gamma$ depending only upon the data and $k_{0}$ such that if $Z_{0}<v$ either $\mu_{+}-\mu_{-} \leq \gamma R_{0}^{N}$ or $\left\{Z_{j}\right\}$ converges to zero as $j$ goes to infinity.

Proof. Define

$$
Q_{R_{0}, j}^{*}:=\left\{x:\left|x_{i}\right|<R_{j}, \forall i=1, \ldots, N-1,\left|x_{N}\right|<R_{0}\right\},
$$

where $R_{j}=\frac{R_{0}}{2}+\frac{R_{0}}{2^{j+1}}$.

Let $\tilde{c}_{j}=\left|Q_{R_{0}, j}^{*}\right|$. Clearly $\tilde{c}_{j}$ is a decreasing sequence converging to a strictly positive constant $c_{\infty}$.

Define

$$
A_{s, j}:=\left\{x \in Q_{R_{0, s}}^{*}: u(x) \leq k_{j}\right\}, \quad k_{j}=\frac{k_{0}}{2}+\frac{k_{0}}{2^{j+1}}+\mu_{-} .
$$

and $Z_{j}:=\frac{\left|A_{2 j, j}\right|}{\left|Q_{R_{0,2 j}}^{*}\right|}$. 
In the sets $Q_{R_{0}, j}^{*}$, choosing as test function $-\theta^{q}\left(u-k_{i}\right)_{-}=-\theta^{q}\left(k_{i}-u\right)_{+}$where $\theta$ does not depend on $x_{N}$, the Caccioppoli generalised inequality becomes

$$
\begin{aligned}
& \int_{Q_{R_{0}, j}^{*}} \theta^{q}\left(\left|D_{x}\left(u-k_{i}\right)_{-}\right|^{q}+\left|D_{x_{N}}\left(u-k_{i}\right)_{-}\right|^{p}\right) d x \\
& \leq C \int_{Q_{R_{0}, j}^{*}}\left|D_{x} \theta\right|^{q}\left|\left(u-k_{i}\right)_{-}\right|^{q} d x
\end{aligned}
$$

where with $D_{x}$ we denoted the gradient with respect the first $N-1$ variables.

On the other hand

$$
\begin{aligned}
& \int_{Q_{R_{0}, j}^{*}} \theta^{q}\left(\left|D_{x}\left(u-k_{i}\right)_{-}\right|^{q}+\left|D_{x_{N}}\left(u-k_{i}\right)_{-}\right|^{q}\right) d x \\
& \leq \int_{Q_{R_{0}, j}^{*}} \theta^{q}\left(\left|D_{x}\left(u-k_{i}\right)_{-}\right|^{q}+\left|D_{x_{N}}\left(u-k_{i}\right)_{-}\right|^{p}\right) d x+\left|A_{j, i}\right|
\end{aligned}
$$

Hence

$$
\begin{aligned}
& \int_{Q_{R_{0}, j}^{*}} \theta^{q}\left(\left|D_{x}\left(u-k_{i}\right)_{-}\right|^{q}+\left|D_{x_{N}}\left(u-k_{i}\right)_{-}\right|^{q}\right) d x \\
& \leq C \int_{Q_{R_{0}, j}^{*}}\left|D_{x} \theta\right|^{q}\left|\left(u-k_{i}\right)_{-}\right|^{q} d x+\left|A_{j, i}\right|
\end{aligned}
$$

The statement, now, follows by the classical properties of non homogeneous DeGiorgi classes, by repeating in a straightforward way the proof of Proposition 4.1, Chapter 10 of [6].

Before stating the last preliminary result, let us introduce some notation. For a function $v$ defined in a set $E$ and real numbers $k<l$, put

$$
\begin{aligned}
{[v>l] } & =\{x \in E \mid v(x)>ł\} \\
{[v<k] } & =\{x \in E \mid v(x)<k\} \\
{[k<v<\ell] } & =\{x \in E \mid k<v(x)<l\} .
\end{aligned}
$$

For $\rho>0$ and $y \in \mathbb{R}^{N}$, denote by $B_{\rho}(y)$ the ball of radius $\rho$ centered at $y$, and by $K_{\rho}(y)$ the cube of edge $\rho$, centered at $y$ and with faces parallel to the coordinate planes. If $y$ is the origin, let $B_{\rho}(0)=B_{\rho}$, and $K_{\rho}(0)=K_{\rho}$.

Lemma 2.7. (DeGiorgi [4]) Let $v \in W^{1,1}\left(K_{\rho}(y)\right)$, and let $k<l$ be real numbers. There exists a constant $\gamma$ depending only on $N, p$ and independent of $k, l, v, y, \rho$, such that

$$
(l-k)|[v<k]| \leq \gamma \frac{\rho^{N+1}}{|[v>l]|} \int_{[k<v<l]}|D v| d x .
$$


Remark 2.8. The conclusion of the lemma continues to hold for functions $v \in$ $W^{1,1}(E)$ provided $E$ is convex; in such a case, instead of $\rho^{N+1}$, we have $d^{N+1}$, where $d$ is the diameter of the set $E$.

\section{First alternative}

Without loss of generality (modulo suitable standard homothetical transformations), we may assume to work in the unitary cube $Q_{\frac{1}{2}}$; we may also assume that $0<$ $u<1$ in $Q_{\frac{1}{2}}$. Consider now a slide $S_{N}$ of this cube, $S_{N}=\left\{\left(x_{1}, \ldots, x_{N-1}, x_{N}\right) \in\right.$ $\left.\mathbb{R}^{N}:-\frac{1}{2}<x_{i}<\frac{1}{2}, \forall 1 \leq i \leq N-1,-\varepsilon<x_{N}<\varepsilon\right\}$ with $\varepsilon$ a small positive number to be quantified later. By using a suitable homothetical transformation, we can transform $S_{N}$ into $T_{N}$ where the set $T_{N}=\left\{\left(x_{1}, \ldots, x_{N-1}, x_{N}\right) \in \mathbb{R}^{N}\right.$ : $\left.-\frac{1}{2} \theta<x_{i}<\frac{1}{2} \theta, \forall 1 \leq i \leq N-1,-\frac{1}{2}<x_{N}<\frac{1}{2}\right\}$ where $\theta$ is a very large positive parameter to be chosen. Note that, when $\theta$ is chosen, $\varepsilon$ too is quantified. Without loss of generality, we may assume $\theta \in \mathbb{N}$. Now, partition $T_{N}$ in sub-cubes $Q^{(j)}$ of unitary wedge.

Assume that for each $Q^{(j)}$

$$
\left|\left[x \in Q^{(j)}: u(x) \leq \frac{1}{2}\right]\right|>v\left|Q^{(j)}\right|
$$

where $v$ is the quantity claimed in Lemma 2.5 .

Lemma 3.1. (The first alternative) Let $u$ be a bounded weak solution of (1) in $Q_{\frac{1}{2}}$. Assume that the structure conditions (2) are satisfied and that (14) holds for any $Q^{(j)}$. Then there is a $n_{0} \in \mathbb{N}$ such that $u \leq 1-\frac{1}{2^{n_{o}+1}}$ for a.e. $x \in \frac{1}{2} T_{N}$.

Proof. Apply Lemma 2.7 to any $Q^{(j)}$ with

$$
l=1-\frac{1}{2^{n}}, \quad k=1-\frac{1}{2^{n-1}}, \quad h=l-k=\frac{1}{2^{n}}, \quad n=2, \ldots, n_{o},
$$

where $n_{o}$ is to be chosen. We obtain

$$
\frac{1}{2^{n}}\left|\left[u>1-\frac{1}{2^{n}}\right] \cap Q^{(j)}\right| \leq \gamma \int_{[k<v<l] \cap Q^{(j)}}|D u| d x .
$$

If we sum over all the $Q^{(j)}$, we get

$$
\frac{1}{2^{n}}\left|\left[u>1-\frac{1}{2^{n}}\right] \cap T_{N}\right| \leq \gamma \int_{[k<v<l] \cap T_{N}}|D u| d x .
$$

Since

$$
\int_{[k<v<l] \cap T_{N}}|D u| d x \leq \int_{[k<v<l] \cap T_{N}}\left|D_{x} u\right| d x+\int_{[k<v<l] \cap T_{N}}\left|D_{x_{N}} u\right| d x,
$$


(where with $D_{x}$ we have denoted the gradient with respect the first $N-1$ variables), denoting $\left[u>1-\frac{1}{2^{n}}\right] \cap T_{N}$ by $A_{n}$, we have

$$
\begin{aligned}
\frac{1}{2^{n}}\left|A_{n}\right| & \leq \gamma \int_{[k<v<l] \cap T_{n}}|D u| d x \\
& \leq \gamma\left(\int_{[k<v<l] \cap T_{N}}\left|D_{x} u\right|^{q} d x\right)^{\frac{1}{q}}\left|A_{n-1} \backslash A_{n}\right|^{\frac{q-1}{q}} \\
& +\gamma\left(\int_{[k<v<l] \cap T_{N}}\left|D_{x_{N}} u\right|^{p} d x\right)^{\frac{1}{p}}\left|A_{n-1} \backslash A_{n}\right|^{\frac{p-1}{p}} .
\end{aligned}
$$

By generalised Caccioppoli inequality applied to $(u-k)_{+}$it follows

$$
\begin{aligned}
& \frac{1}{2^{n}}\left|A_{n}\right| \\
& \leq \gamma\left[\int _ { 2 T _ { N } } \left[\zeta^{p-q}\left|D_{x} \zeta\right|^{q}\left|(u-k)_{+}\right|^{q}\right.\right. \\
& \left.\left.\quad+\left|D_{x_{N}} \zeta\right|^{p}\left|(u-k)_{+}\right|^{p}\right] d x\right]^{\frac{1}{q}} \cdot\left|A_{n-1} \backslash A_{n}\right|^{\frac{q-1}{q}} \\
& \quad+\gamma\left[\int _ { 2 T _ { N } } \left[\zeta^{p-q}\left|D_{x} \zeta\right|^{q}\left|(u-k)_{+}\right|^{q}\right.\right. \\
& \left.\left.\quad+\left|D_{x_{N}} \zeta\right|^{p}\left|(u-k)_{+}\right|^{p}\right] d x\right]^{\frac{1}{p}} \cdot\left|A_{n-1} \backslash A_{n}\right|^{\frac{p-1}{p}}
\end{aligned}
$$

By choosing a suitable $\zeta$

$$
\begin{aligned}
& \frac{1}{2^{n}}\left|A_{n}\right| \\
& \quad \leq \gamma\left[h^{p}\left(\frac{1}{\theta^{q} h^{p-q}}+1\right)\left|T_{N}\right|\right]^{\frac{1}{q}} \cdot\left|A_{n-1} \backslash A_{n}\right|^{\frac{q-1}{q}} \\
& \quad+\gamma\left[h^{p}\left(\frac{1}{\theta^{q} h^{p-q}}+1\right)\left|T_{N}\right|\right]^{\frac{1}{p}} \cdot\left|A_{n-1} \backslash A_{n}\right|^{\frac{p-1}{p}},
\end{aligned}
$$

since by the choice of $k$ and $h$ we have $(u-k)_{+}<1-\left(1-\frac{1}{2^{n-1}}\right)<2 h$. Moreover

$$
\begin{aligned}
& \frac{1}{2^{n}}\left|A_{n}\right| \\
& \quad \leq \gamma\left(\frac{1}{2^{n}}\right)^{\frac{p}{q}}\left[\left(\frac{1}{\theta^{q} h^{p-q}}+1\right)\left|T_{N}\right|\right]^{\frac{1}{q}} \cdot\left|A_{n-1} \backslash A_{n}\right|^{\frac{q-1}{q}} \\
& \quad+\gamma\left(\frac{1}{2^{n}}\right)\left[\left(\frac{1}{\theta^{q} h^{p-q}}+1\right)\left|T_{N}\right|\right]^{\frac{1}{p}} \cdot\left|A_{n-1} \backslash A_{n}\right|^{\frac{p-1}{p}}
\end{aligned}
$$


Suppose that $n_{o}$ has already been chosen, and let

$$
\theta=2^{n_{o} \frac{p-q}{q}}
$$

As mentioned above, provided $n_{o}$ is sufficiently large, we can always assume that $n_{o} \frac{p-q}{q}$ is an integer. Due to the previous choice for $\theta$ we derive

$$
\left|A_{n}\right| \leq \gamma\left(\left|T_{N}\right|^{\frac{1}{p}}\left|A_{n-1} \backslash A_{n}\right|^{\frac{p-1}{p}}+\left|T_{N}\right|^{\frac{1}{q}}\left|A_{n-1} \backslash A_{n}\right|^{\frac{q-1}{q}}\right) .
$$

At each step,we have that one of these two inequalities holds:

$$
\left\{\begin{array}{l}
\left|A_{n}\right|^{\frac{p}{p-1}} \leq \gamma|Q|^{\frac{1}{p-1}}\left|A_{n-1} \backslash A_{n}\right| \\
\text { or } \\
\left|A_{n}\right|^{\frac{q}{q-1}} \leq \gamma|Q|^{\frac{1}{q-1}}\left|A_{n-1} \backslash A_{n}\right| .
\end{array}\right.
$$

Once more, suppose that $n_{o}$ has already been chosen: then, or for $p$ or for $q$ the previous inequality holds at least $\frac{n_{o}}{2}$ times. Therefore, summing $A_{n}$ in (15) when it holds for $p$ or $q$, we conclude that

$$
\frac{n_{o}}{2}\left|\left[x \in T_{N}: u(x) \geq 1-\frac{1}{2^{n_{o}}}\right]\right|<\gamma\left|T_{N}\right| .
$$

The value of $n_{o}$ is determined in such a way that

$$
\frac{2 \gamma}{n_{o}} \leq v
$$

where $v$ is the same parameter as in Lemma 2.5. Notice that $T_{N}$ is scaled correctly according to the intrinsic geometry considered in Lemma 2.5. Therefore, we can apply such a Lemma, and conclude that

$$
u \leq 1-\frac{1}{2^{n_{o}+1}}
$$

for a.e. $x \in \frac{1}{2} T_{N}$.

Hence, if the first alternative occurs, we have reduced the oscillation of the solution $u$ in a quantitative way in $S_{N}$.

\section{Second alternative and Proof of Theorem 1.1}

If the first alternative does not occur, there exists at least one $Q^{(j)} \subset T_{N}$, centered at $x_{0}=\left(x_{1}^{(j)}, \ldots, x_{N-1}^{(j)}, 0\right)$, such that

$$
\left|\left[x \in Q^{(j)}: u(x) \leq \frac{1}{2}\right]\right| \leq v\left|Q^{(j)}\right| .
$$

Without loss of generality we may assume $x_{0}=\left(x_{1}^{(j)}, 0,0, \ldots, 0\right)$. Define $Q^{0}$ the cube centered in the origin, so $Q^{(j)}=x_{0}+Q^{0}$. 
In the next lemma, we apply the logarithmic estimates to expand the positivity of $Q^{(j)}$ till the origin.

Apply Lemma 2.5 to $Q^{(j)}$ to get, for a.e. $x \in \frac{1}{2} Q^{(j)}$

$$
u(x) \geq \frac{1}{4} .
$$

Lemma 4.1. (The second alternative) Let $u$ be a bounded weak solution of (1) in $Q_{\frac{1}{2}}$. Assume that conditions (2) are satisfied and that (14) does not hold for a $Q^{(j)}$. Then for any positive constant $\nu_{0} \in(0,1)$, there exists a positive integer $s_{0}$ such that

$$
\mid\left\{x \in \frac{1}{2} Q^{0}: u(x) \leq e^{-s_{0}}\left|\leq v_{0}\right| \frac{1}{2} Q^{0} \mid .\right.
$$

Proof. If we perform the change of variable

$$
\begin{aligned}
& x_{1}-x_{1}^{(j)}=\frac{y_{1}}{2} \\
& x_{i}=\frac{y_{i}}{2}, \quad \forall i=2, \ldots, N-1 \\
& x_{N}=\left(\frac{1}{4}\right)^{\frac{p-q}{p}} \frac{y_{N}}{2}
\end{aligned}
$$

and calling $v=4 u$, you have that $v$ satisfies an equation of the type (1) satisfying (2). Moreover $v \geq 1$ in the unitary cube centered in the origin. As already noticed, the Lemma is proved if we are able to transport some positivity till to the point $z=\left(-2 x_{1}^{(j)}, 0, \ldots, 0\right)$.

Let us denote the solution $v$ with $u$ and the coordinates $\left(y_{1}, \ldots, y_{N}\right)$ with $\left(x_{1}, \ldots, x_{N}\right)$. Without loss of generality assume $-2 x_{1}^{(j)}>0$, denote with $L=$ $-2 x_{1}^{(j)}$ and assume $L>1$.

Define $S$ the slide of the half unitary cube centered in the origin in the $x^{\prime}=$ $\left(x_{2}, \ldots, x_{N}\right)$ variables, i.e $\left\{x \in \mathbb{R}^{\mathbb{N}}: x=\left(0, x_{2}, \ldots, x_{N}\right):-\frac{1}{4}<x_{i}<\frac{1}{4}, \forall i=\right.$ $2, \ldots, N\}$.

Let $s_{0}>1$ be an integer, to be fixed later, and define a set $A$ in $S$ so defined:

$$
A=\left\{\left(0, x^{\prime}\right) \in S: \exists t \in[0,2 L], u\left(t, x^{\prime}\right) \leq e^{-s_{0}}\right\}
$$

We want to prove that for any positive constant $v_{0} \in(0,1)$, there exists a positive integer $s_{0}$ such that

$$
\frac{|A|}{|S|} \leq v_{0} .
$$

Let $x^{\prime} \in A$. Then $\exists t \in[0,2 L]$ such that $u\left(t, x^{\prime}\right) \leq e^{-s_{0}}$.

Therefore,

$$
\begin{aligned}
s_{0}-1 & \leq \ln _{+} \frac{u\left(0, x^{\prime}\right)+e^{-s_{0}}}{u\left(t, x^{\prime}\right)+e^{-s_{0}}} \\
& =\ln _{+} \frac{1}{u\left(t, x^{\prime}\right)+e^{-s_{0}}}-\ln _{+} \frac{1}{u\left(0, x^{\prime}\right)+e^{-s_{0}}} \\
& =\int_{0}^{t} D_{x_{1}}\left(\ln _{+} \frac{1}{u\left(s, x^{\prime}\right)+e^{-s_{0}}}\right) d s \leq \int_{0}^{2 L}\left|D_{x_{1}}\left(\ln _{+} \frac{1}{u\left(s, x^{\prime}\right)+e^{-s_{0}}}\right)\right| d s
\end{aligned}
$$


Now if we integrate the inequality above over the set $A$, we obtain

$$
\left(s_{0}-1\right)|A| \leq \int_{S} \int_{0}^{2 L}\left|D_{x_{1}}\left(\ln _{+} \frac{1}{u(x)+e^{-s_{0}}}\right)\right| d x
$$

Using Hölder Inequality, we have

$$
\left(s_{0}-1\right)|A| \leq\left[\int_{S} \int_{0}^{2 L}\left|D_{x_{1}} \ln _{+}\left(\frac{1}{u(x)+e^{-s_{0}}}\right)\right|^{q} d x\right]^{\frac{1}{q}}[2 L|S|]^{\frac{q-1}{q}} .
$$

Here if we consider Lemma 2.1 choosing $\xi=1$ in $[0,2 L] \times S$ and $\xi=0$ out of $[0,3 L] \times 2 S$, and putting $H \omega=1$ and $a=e^{-s_{0}}$ we get

$$
\begin{aligned}
\left(s_{0}-1\right)|A| \leq C & {\left[\int_{2 S} \int_{0}^{3 L} \sum_{i=1}^{N-1}\left|D_{x_{i}} \xi\right|^{q} d x\right.} \\
& \left.+\iint_{2 S}^{3 L}\left|D_{x_{N}} \xi\right|^{p} e^{-s_{0}(p-q)} d x\right]^{\frac{1}{q}}[2 L|S|]^{\frac{q-1}{q}} . \\
\left(s_{0}-1\right)|A| \leq C L|S| &
\end{aligned}
$$

Dividing by $|S|$ we have

$$
\frac{|A|}{|S|} \leq C \frac{L}{s_{0}-1}
$$

and (17) follows choosing $s_{0}$ large enough, and (17), in turn, implies (16).

Thanks to the previous result, we have proved that, around the origin, the solution is far away from zero in most of the cube. However the geometry is not the intrinsic one, so we cannot apply directly the De Giorgi- type lemma. To overcome this difficulty we find two slides, one up and the other one down the origin in the $x_{N}$ variable, where the solution is far away from zero everywhere. Now we are in the condition to apply Lemma 2.6 and get the positivity everywhere inside the two slides.

Let us prove the main result of this note.

Proof of Theorem 1.1. By using the same notation of Lemma 4.1, choose $v_{0}=$ $\frac{1}{4^{N}} \frac{v}{10}$, where $v$ is the small constant defined in Lemma 2.5, where $R_{N}=R_{0}^{\frac{q}{p}} k_{0}^{\frac{p-q}{p}}$. Let $P$ be the parallelepiped having the first $N-1$ sides long $\frac{1}{2}$ and the last one $\left(\frac{1}{2}\right)^{\frac{q}{p}} e^{-s_{0} \frac{p-q}{p}}$.

With this choice there exists a $x_{0+}^{N} \in\left[\frac{1}{8}, \frac{1}{4}\right]$ such that in the set

$$
R_{L}:=\left(L, 0, \ldots, 0, x_{N}^{0+}\right)+P
$$


the measure where $u$ is smaller than $e^{-s_{0}}$ is smaller than $v|P|$. If such thing was not occurring, then, in the parallelepiped $P^{\diamond}=\left[L-\frac{1}{4}, L+\frac{1}{4}\right] \times\left[-\frac{1}{4}, \frac{1}{4}\right]^{N-2} \times$ $\left[\frac{1}{8}, \frac{1}{4}\right]$, the measure where $u$ is smaller than $e^{-s_{0}}$ would be larger than $v\left|P^{\diamond}\right|$ and this is in contradiction with (16) and the choice $v_{0}=\frac{1}{4^{N}} \frac{v}{10}$. Therefore by Lemma 2.5. in the set $\left(L, 0, \ldots, 0, x_{0+}^{N}\right)+\frac{1}{2} P$ we have $u \geq \frac{1}{2} e^{-s_{0}}$.

Analogously there exists $x_{0-}^{N} \in\left[-\frac{1}{4},-\frac{1}{8}\right]$ such that in the set $\left(L, 0, \ldots, 0, x_{0-}^{N}\right)+$ $\frac{1}{2} P$ we have $u \geq \frac{1}{2} e^{-s_{0}}$.

Therefore in the parallelepiped $P_{1}=\left\{x \in \mathbb{R}^{N}: L-\frac{1}{4}<x_{1}<L+\frac{1}{4},-\frac{1}{4}<\right.$ $\left.x_{2}<\frac{1}{4}, \ldots,-\frac{1}{4}<x_{N-1}<\frac{1}{4}, x_{0-}^{N}<x_{N}<x_{0+}^{N}\right\}$ we have that on the faces $x_{N}=x_{0-}^{N}$ and $x_{N}=x_{0+}^{N}$ the solution $u$ is bigger or equal than $\frac{1}{2} e^{-s_{0}}$. Moreover, by the choice of $v_{0}$, analogously as above, one can prove that the measure where $u<e^{-s_{0}}$ is smaller than $\nu\left|P_{1}\right|$. Therefore, the assumptions of Lemma 2.6 are fulfilled and we have either $u \geq \frac{1}{4} e^{-s_{0}}$ in $\frac{1}{2} P_{1}$ or the oscillation is quantitatively small.

Hence, also in the case of the second alternative, we reduced the oscillation of the solution $u$ in a quantitative way and this implies the Hölder continuity of the solution.

Acknowledgements The authors are members of the Gruppo Nazionale per l'Analisi Matematica, la Probabilità e le loro Applicazioni (GNAMPA) of the Istituto Nazionale di Alta Matematica (INdAM). We want also to thank the anonymous referee for the careful reading of the paper and for the valuable suggestions.

\section{References}

[1] Boccardo, L., Marcellini, P., Sbordone, C.: $L^{\infty}$-regularity for variational problems with sharp nonstandard growth conditions. Boll. Un. Mat. Ital. 4-A, 219-226 (1990)

[2] Cupini, G., Marcellini, P., Mascolo, E.: Regularity under sharp anisotropic general growth conditions. Discrete Contin. Dyn. Syst. Ser. B 11, 66-86 (2009)

[3] Cupini, G., Marcellini, P., Mascolo, E.: Local boundedness of solutions to quasilinear elliptic systems. Manuscr. Math. 137, 287-315 (2012)

[4] De Giorgi, E.: Sulla differenziabilità e l'analiticità delle estremali degli integrali multipli regolari. Mem. Accad. Sci. Torino. Cl. Sci. Fis. Mat. Nat. (3) 3, 25-43 (1957)

[5] DiBenedetto, E.: Degenerate Parabolic Equations. Springer, New York (1993)

[6] DiBenedetto, E.: Partial Differential Equations, Second Edition (Cornerstones). Birkhuser Boston Inc., Boston (2010)

[7] DiBenedetto, E., Gianazza, U., Vespri, V.: Harnack estimates for quasi-linear degenerate parabolic differential equations. Acta Math. 200, 181-209 (2008)

[8] DiBenedetto, E., Gianazza, U., Vespri, V.: Harnack's Inequality for Degenerate and Singular Parabolic Equations (Springer Monographs in Mathematics). Springer, New York (2012)

[9] DiBenedetto, E., Gianazza, U., Vespri, V.: Remarks on local boundedness and local Hölder continuity of local weak solutions to anisotropic p-Laplacian type equations. J. Elliptic Parabol. Equ. 2(1-2), 157-169 (2016)

[10] Düzgün, F.G., Gianazza, U., Vespri, V.: 1-dimensional Harnack estimates. Discrete Contin. Dyn. Syst. Ser. S 9, 675-685 (2016) 
[11] Düzgün, F.G., Marcellini, P., Vespri, V.: Space expansion for a solution of an anisotropic $p$-Laplacian equation by using a parabolic approach. Riv. Math. Univ. Parma 5, 93-111 (2014)

[12] Düzgün, F.G., Marcellini, P., Vespri, V.: An alternative approach to the regularity of degenerate elliptic equations. Nonlinear Anal. 94, 133-141 (2014)

[13] Giaquinta, M.: Growth conditions and regularity, a counter example. Manuscr. Math. 59, 245-248 (1987)

[14] Kolodī̌̆, İ.M.: The boundedness of generalized solutions of elliptic differential equations, Vestnik Moskov. Univ. Ser. I Mat. Meh. 25, 44-52 (1970) (Russian). English transl. Moscow Univ. Math. Bull. 25, 31-37 (1970)

[15] Liskevich, V., Skrypnik, I.I.: Hölder continuity of solutions to an anisotropic elliptic equation. Nonlinear Anal. 71, 1699-1708 (2009)

[16] Marcellini, P.: Regularity of minimizers of integrals of the calculus of variations with nonstandard growth conditions. Arch. Ration. Mech. Anal. 105, 267-284 (1989)

[17] Marcellini, P.: Regularity and existence of solutions of elliptic equations with $p, q$ growth conditions. J. Differ. Equ. 90, 1-30 (1991)

[18] Marcellini, P.: Regularity for some scalar variational problems under general growth conditions. J. Optim. Theory Appl. 90, 161-181 (1996)

[19] Mingione, G.: Regularity of minima: an invitation to the dark side of the calculus of variations. Appl. Math. 51(4), 355-426 (2006)

[20] Nikolskii, S.M.: Imbedding theorems for functions with partial derivatives considered in various metrics. Izd. Akad. Nauk. SSSR 22, 321-336 (1958). English trans.: Am. Math. Soc. Trans. 90, 27-44 (1970)

[21] Troisi, M.: Teoremi di inclusione per spazi di Sobolev non isotropi. Ricerche Mat. 18, 3-24 (1969)

[22] Urbano, J.M.: The Method of Intrinsic Scaling: A Systematic Approach to Regularity for Degenerate and Singular PDEs, Lecture Notes in Mathematics 1930. Springer, Berlin, Heidelberg (2008) 